\title{
Membranas cerâmicas assimétricas tubulares: influência do tempo de deposição da camada filtrante na morfologia das membranas
}

\author{
Asymmetric tubular ceramic membrane: \\ influence of the filter layer deposition \\ time on the membrane morphology
}

\author{
Mirele Costa da Silva ${ }^{1}$, Daniel Silveira Lira ${ }^{2}$, \\ Normanda Lino de Freitas ${ }^{3}$, Helio Lucena Lira ${ }^{4}$
}

\footnotetext{
${ }^{1}$ Laboratório de membranas - Labcem - UFCG - CEP: 58.429-900, Campina Grande, PB

e-mail: mirelecsilva@hotmal.com; daniellira@hotmail.com; normanda@dema.ufcg.edu.br; helio@dema.ufcg.edu.br
}

\begin{abstract}
RESUMO
Esse trabalho tem como objetivo obter membranas cerâmicas assimétricas para aplicação em processos de microfiltração e avaliar a influência do tempo de deposição (5 e 10 s) de uma dispersão de argila sobre um suporte tubular de alumina comercial e bentonita. O suporte tubular foi caracterizado por microscopia ótica e eletrônica de varredura e por porosimetria. A membrana assimétrica foi caracterizada por microscopia eletrônica de varredura e por porosimetria. Os resultados mostraram suportes porosos com espessura de $1092 \mu \mathrm{m}$, diâmetro médio de poro de 0,99 $\mu \mathrm{m}$ e porosidade de 39\%. A membrana assimétrica foi obtida com sucesso para os tempos de deposição avaliados, sendo classificada para aplicações em processos de microfiltração atingindo diâmetro médio de poro de $0,25 \mu \mathrm{m}$ e porosidade de $35 \%$.
\end{abstract}

Palavras-chave: membrana assimétrica, membrana tubular, deposição, microfiltração.

\section{ABSTRACT}

This paper aims to obtain asymmetric ceramic membranes for application in microfiltration processes and evaluate the influence of the deposition time (5 and $10 \mathrm{~s}$ ) of a clay dispersion on commercial alumina and bentonite tubular support. The tubular support was characterized by optical and scanning electron microscopy and porosimetry. The asymmetric membrane was characterized by scanning electron microscopy and porosimetry. The results showed porous substrates with thickness of $1092 \mu \mathrm{m}$, average pore diameter of 0.99 $\mu \mathrm{m}$ and porosity of $39 \%$. The asymmetric membrane was successfully obtained for the evaluated deposition times, being classified for microfiltration processes applications.

Keywords: asymmetric membrane, tubular membrane, deposition, microfiltration.

\section{INTRODUÇÃO}

A tecnologia de separação com membranas tem sido amplamente utilizada em biotecnologia, indústrias farmacêuticas e de alimentos ou para tratar outros efluentes industriais. Essa tecnologia oferece uma ampla gama de procedimentos capazes de recuperar, concentrar ou purificar solventes valiosos em todos estes campos científicos [1].

As membranas inorgânicas apresentam vantagens sobre as orgânicas por poderem atuar sob condições em que as orgânicas apresentam restrições, como por exemplo, na presença de solventes, elevadas temperaturas e pressões. Procedimentos agressivos de limpeza química e/ou mecânica e esterilização são geralmente empregados em membranas e são propensos a causar danos na estrutura da membrana especialmente para as membranas orgânicas [2]. Entretanto, as membranas inorgânicas são normalmente mais caras do que as orgânicas habituais. Nesse sentido, novas pesquisas com membranas usando materiais cerâmicos de custo mais baixo devem difundir o uso da tecnologia de membranas, especialmente para países emergentes, onde muitos problemas ambientais podem ser resolvidos com a utilização de membranas. 
Membranas cerâmicas são normalmente preparadas a partir de óxidos como alumina, zircônia e titânia, o que eleva o custo de produção. Dessa forma, pesquisas vêm sendo desenvolvidas na busca de minimizar esses custos e uma das formas para isto é a utilização de matérias-primas naturais de baixo custo. A literatura tem apresentado resultados significativos quando utilizadas argilas como matéria-prima para confecção de membranas. Palacio et al. [1] confeccionaram membranas cerâmicas de microfiltração a partir de argilas e fosfatos vindos de minérios marroquinos. Os resultados demonstraram ser bastante promissores para serem utilizados como um passo de clarificação prévia no tratamento de águas de indústrias têxteis. Khemakhem e Amar [3] obtiveram membranas cerâmicas de argila para aplicação em processos de destilação. Em seus resultados os autores atingiram taxas de rejeição elevada de sal superior a 99\%. Khemakhem et al. [4] prepararam membranas para microfiltração com argila usadas para tratar efluente gerado a partir do condicionamento (congelamento e lavagem) de indústria de alimentos do mar. Os autores mostraram que as membranas preparadas são adequadas para o tratamento dessas águas residuais. Vercauteren et al. [5] prepararam membranas cerâmicas de multicamadas com a deposição da camada de argila por imersão em uma membrana de $\gamma$ alumina numa suspensão de montmorilonita. Em seus resultados os autores mostraram, com experiências preliminares, uma resistência ao fluxo elevada para a permeação de gases individuais, possivelmente devido a uma polarização incompleta do filme depositado de argila.

Dentre as morfologias apresentadas pelas membranas, a assimétrica, cuja membrana é composta por um suporte poroso, por uma ou várias camadas intermediárias e pela camada filtrante, se destaca por atingir maiores fluxos devido à camada filtrante ser muito fina. Uma estrutura de múltiplas camadas pode ser obtida pela repetição do procedimento de revestimento, geralmente incluindo o passo de sinterização e usando as condições adaptadas para cada camada de revestimento [ 6$]$.

Diante do exposto, esse trabalho tem como objetivo a obtenção de membrana cerâmica assimétrica tubular para aplicações em processos de microfiltração e a realização da avaliação da influência do tempo de deposição da dispersão utilizada na formação da camada filtrante na morfologia da membrana.

\section{MATERIAIS E MÉTODOS}

Para confecção dos suportes foram utilizados como matérias-primas uma alumina comercial da ALCOA, bentonita proveniente da cidade de Boa Vista no Estado da Paraíba, amido de milho, oleína e água. Para a dispersão com a finalidade de aplicá-la para obtenção da camada filtrante da membrana foi utilizada uma argila caulinítica cedida pela empresa ARMIL.

A massa para confecção dos suportes foi preparada em um misturador através da homogeneização de $90 \%$ de alumina com 10\% de bentonita. Foram adicionados como aditivo: $2 \%$ de oleína, utilizada como lubrificante para auxiliar no processo de extrusão, $2 \%$ de amido de milho, utilizado como ligante e $22 \%$ de água, todos esses percentuais calculados sobre o valor total da massa. A massa cerâmica ilustrou maior percentual de alumina 92\%, atingindo a fase alfa e alguns traços de quartzo [7].

A confecção dos suportes foi realizada em uma extrusora fabricada pela empresa Verdés, modelo 051. Após conformação, os tubos foram submetidos a duas etapas de secagem. A primeira em temperatura ambiente, $25^{\circ} \mathrm{C}$, e uma segunda etapa em estufa nas temperaturas de 40,50 e $60^{\circ} \mathrm{C}$ durante $24 \mathrm{~h}$ para cada temperatura. A queima foi realizada em forno elétrico seguindo os seguintes patamares: de 25 a $700^{\circ} \mathrm{C}$ a uma taxa de aquecimento de $2^{\circ} \mathrm{C} /$ min e de 700 a $1200^{\circ} \mathrm{C}$ com taxa de aquecimento de $5^{\circ} \mathrm{C} /$ min e patamar de $1 \mathrm{~h}$ na temperatura de $1200^{\circ} \mathrm{C}$.

A massa para obtenção da dispersão foi preparada na proporção (80-20)\% de água-massa, com 0,7 g de metacrilato de amônio, utilizado como defloculante. A dispersão foi colocada em um agitador Hamilton beach modelo 930, a uma velocidade de 17000 rpm por 30 min. A massa utilizada para confecção da camada filtrante apresentou maior percentual de óxido de silício, 66\% com a caulinita como fase predominante [7].

A metodologia utilizada para obtenção da camada filtrante da membrana está representada na Figura 1. O suporte foi vedado em uma de suas extremidades e a dispersão foi depositada na região interna do mesmo durante $10 \mathrm{~s}$. A extremidade vedada foi liberada para que o excesso da dispersão escoasse. As peças foram submetidas a duas etapas de secagem, seguida da etapa de queima, utilizando a mesma metodologia descrita para o suporte. 


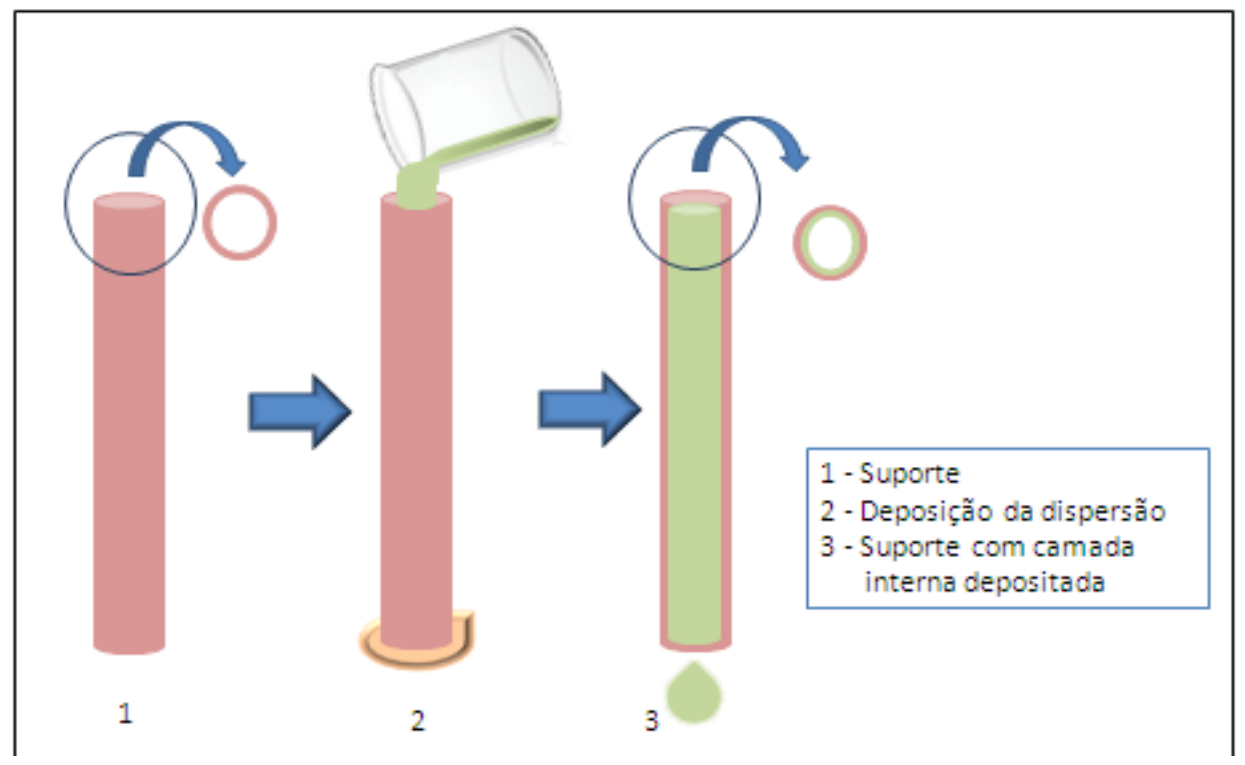

Figura 1: Deposição da dispersão para obtenção da camada filtrante sobre a superfície interna do suporte tubular.

O suporte isolado foi caracterizado por microscopia óptica em um microscópio óptico HIROX de reflexão e transmissão com acessórios 2D e variação de 50-400x, acoplado a uma estação de análise de imagem, por microscopia eletrônica de varredura em um microscópio da Shimadzu, modelo SSX-550 e foi realizada a análise de porosimetria por intrusão ao mercúrio em um porosímetro de mercúrio modelo Autopore IV, da marca Micromeritics. A membrana foi caracterizada por microscopia eletrônica de varredura e por porosimetria por intrusão ao mercúrio nos mesmos equipamentos citados anteriormente.

\section{RESULTADOS}

As imagens obtidas por microscopia óptica após corte transversal no suporte podem ser visualizadas na Figura 2. Com base nas imagens percebe-se que a espessura do suporte tubular atingiu média de $1092 \mu \mathrm{m}$ após média calculada a partir de quatro medições em diferentes áreas da seção transversal. A ampliação da área demonstra nitidamente a estrutura porosa do suporte, entretanto, não é possível, por meio da imagem, identificar a dimensão dos poros. Percebe-se que a estrutura do suporte encontra-se isenta de defeitos, como por exemplo, trincas e/ou rachaduras.

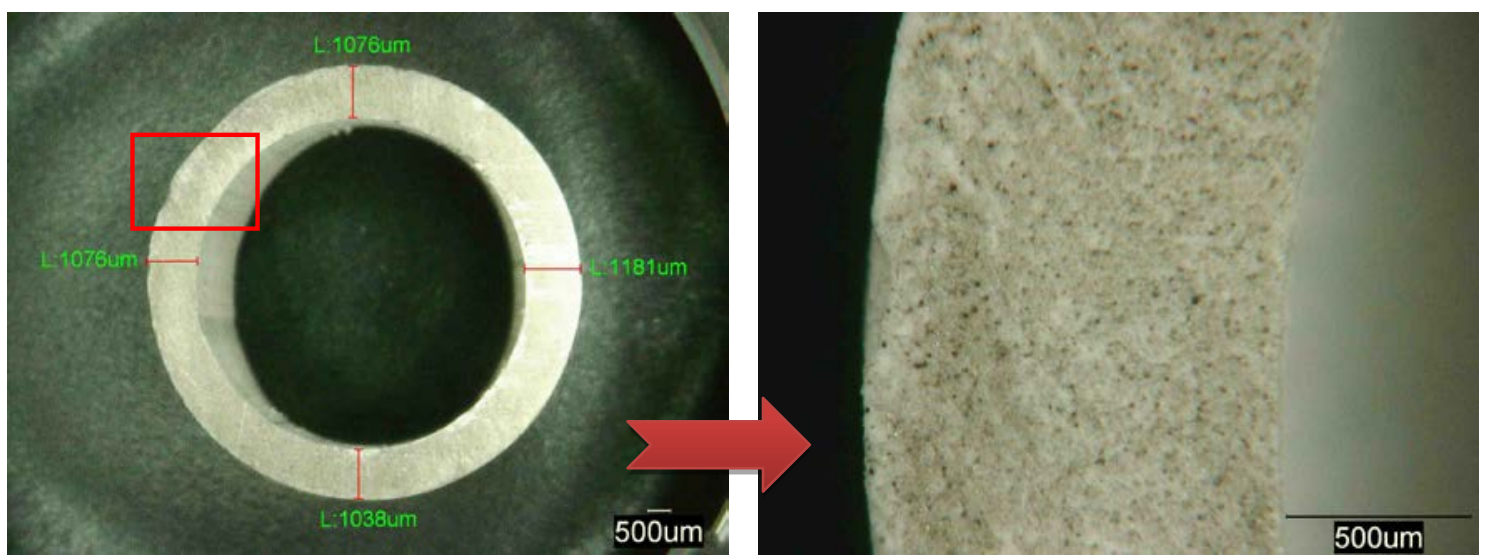

Figura 2: Seção transversal do suporte poroso obtida por microscopia óptica.

Na Figura 3 pode ser observada a morfologia do suporte através da microscopia eletrônica de varredura para a região interna e para a seção transversal. 

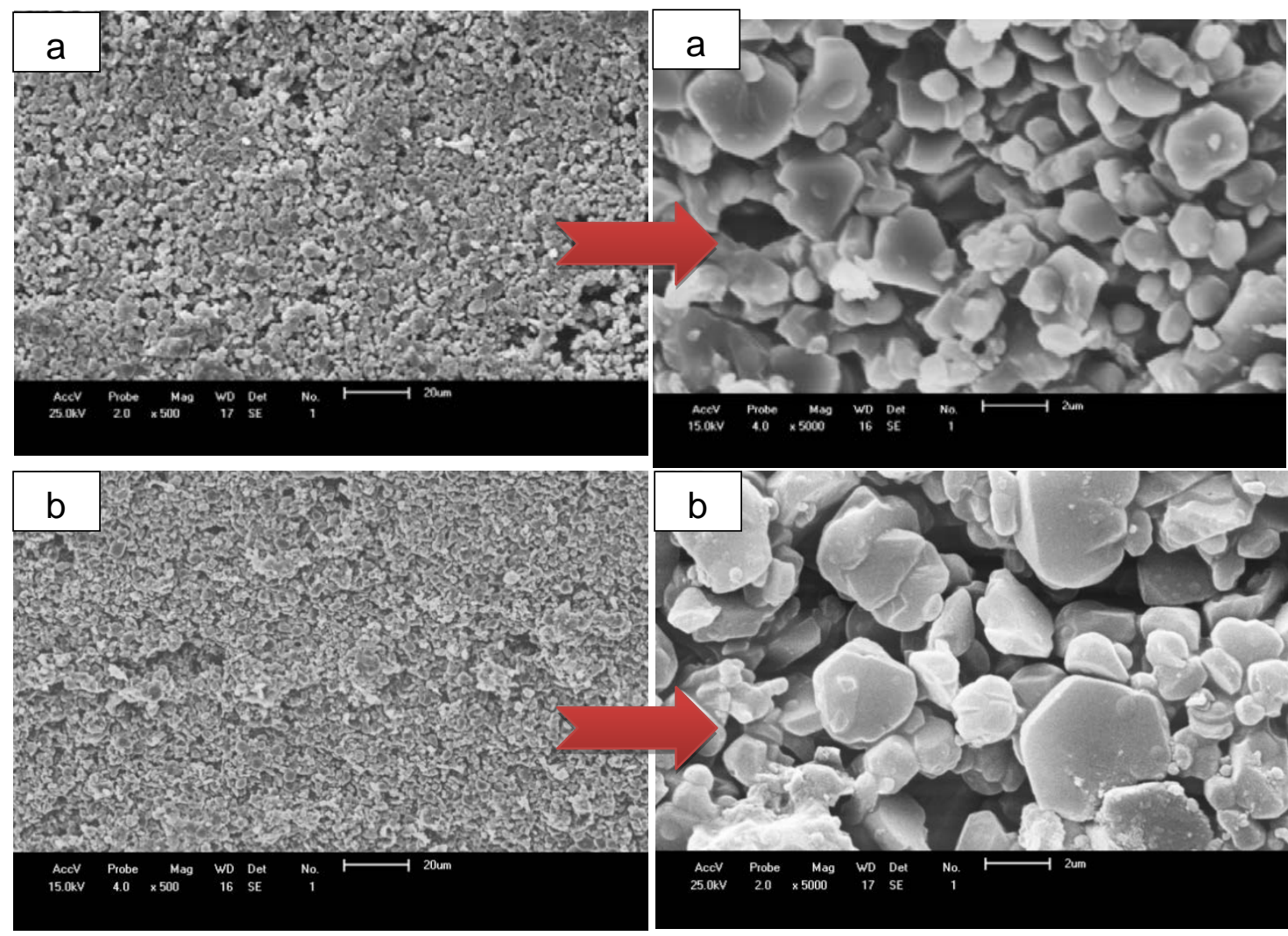

Figura 3: Micrografias do suporte poroso para a região interna (a) e para a seção transversal (b) obtidas em diferentes aumentos.

Observando as micrografias referentes à região interna do suporte, Figura 3a, é possível verificar a presença de poros para todas as áreas analisadas, percebe-se que a mesma está ausente de defeitos superficiais, é notável a presença de partículas apresentando certa esfericidade, característica das partículas de alumina presentes na composição da massa utilizada, com dimensões em sua maioria inferiores a $2 \mu \mathrm{m}$.

Para a seção transversal, Figura 3b, percebe-se a coalescência dos grãos, caracterizando a sinterização da peça cerâmica, assim como é visível a presença de espaços entre os grãos coalecidos o que permite a seletividade da filtração.

As micrografias apresentadas são compatíveis com as micrografias apresentadas na literatura por Silva et al. [ㅁ] que realizaram uma caracterização estrutural de suporte de alumina, antes e depois da deposição de uma película de óxido de titânio na sua superfície para obtenção de membrana assimétrica destinada a aplicação em processos de separação de gás. Cheong et al. [9] ilustraram em sua pesquisa micrografias de membrana de alumina obtida sobre suporte também de alumina e a mesma membrana sem o suporte. Em seus resultados os autores deixaram evidenciado que a melhor distribuição das partículas de alumina na membrana foi atingida quando depositada sobre o suporte.

Conforme referenciado na literatura [6], se os poros forem menores e apresentarem menor rugosidade quando comparados com os da camada filtrante, a estrutura do suporte suprime muito a permeabilidade do sistema. A aplicação de uma ou mais camadas na produção da membrana diminui tanto o tamanho médio dos poros do substrato, como a rugosidade da superfície do substrato e a densidade.

Pode ser observado na Figura 4 a distribuição dos diâmetros dos poros do suporte poroso. 


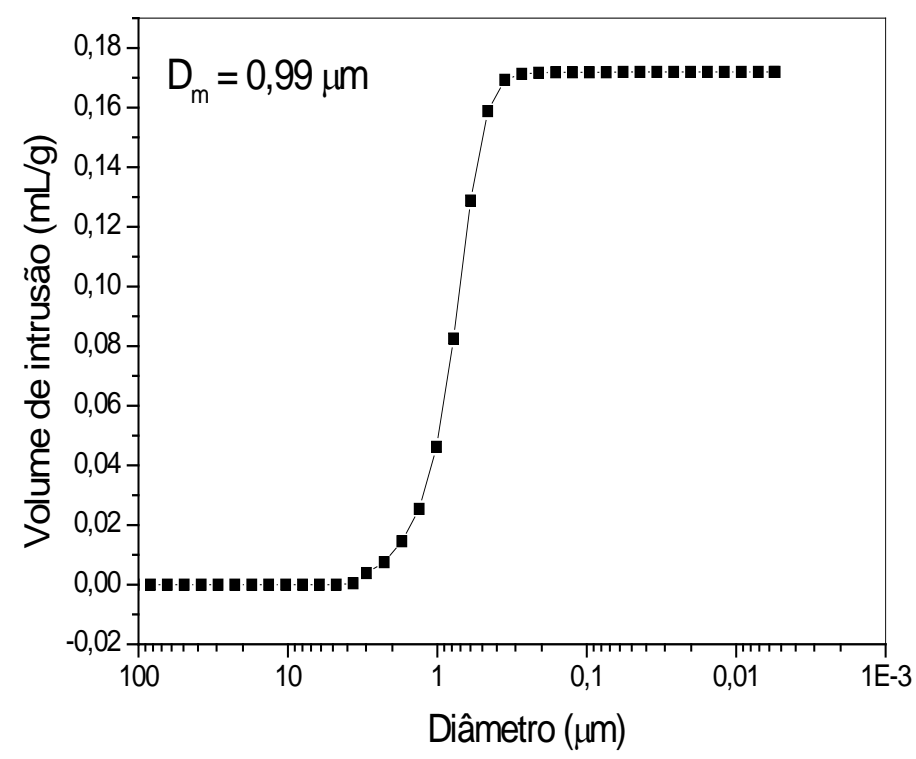

Figura 4: Distribuição dos valores dos diâmetros dos poros do suporte.

O resultado obtido apresenta um suporte com diâmetro médio de poros de 0,99 $\mu \mathrm{m}$ atingindo uma porosidade de 39\%. Com base na curva apresentada percebe-se que a maioria dos valores referentes ao diâmetro médio dos poros estão na faixa de 1,0 a 0,3 $\mu \mathrm{m}$. Comparando o valor alcançado com alguns trabalhos ilustrados na literatura em que pesquisadores utilizaram alumina na obtenção de suportes para fabricar membranas assimétricas, tem-se que Barma e Mandal [10] atingiram diâmetro médio de poro de 0,51 $\mu$ m e porosidade de 55\%, Dong et al. [11] conseguiram chegar a 3,14 $\mu \mathrm{m}$ e Hu et al. [12] atingiram valor bem próximo ao citado anteriormente alcançando 3,3 $\mu \mathrm{m}$.

O valor apresentado para o diâmetro médio de poro serve como base para obtenção da camada subsequente a ser depositada sobre o suporte. O diâmetro das partículas a serem utilizadas para obtenção da camada filtrante não pode atingir dimensão inferior ao diâmetro de poro atingido pelo suporte, nesse caso, 0,99 $\mu \mathrm{m}$. Se essas partículas fossem inferiores ao diâmetro do poro do suporte poderia acarretar o entupimento desses poros, o que diminuiria o fluxo do sistema.

As micrografias das membranas assimétricas obtidas nos tempos de 5 e $10 \mathrm{~s}$ podem ser visualizadas nas Figuras 5 e 6 respectivamente, de deposição da dispersão de argila sobre a superfície interna do suporte poroso.

Mediante as micrografias apresentadas para as membranas, percebe-se que, independente do tempo de deposição utilizado, foi possível a obtenção de uma fina camada filtrante aderida ao suporte poroso.

Para o tempo de 5 s de deposição, percebe-se que a superfície da membrana, representada pelo número 2, apresenta alguns defeitos superficiais e áreas do suporte não recobertas. Nota-se que, para esse tempo de deposição, houve um maior grau de sinterização das partículas e menor porosidade quando comparada a membrana obtida no tempo de $10 \mathrm{~s}$. A superfície da membrana obtida no tempo de $10 \mathrm{~s}$ encontra-se totalmente ausente de defeitos. 


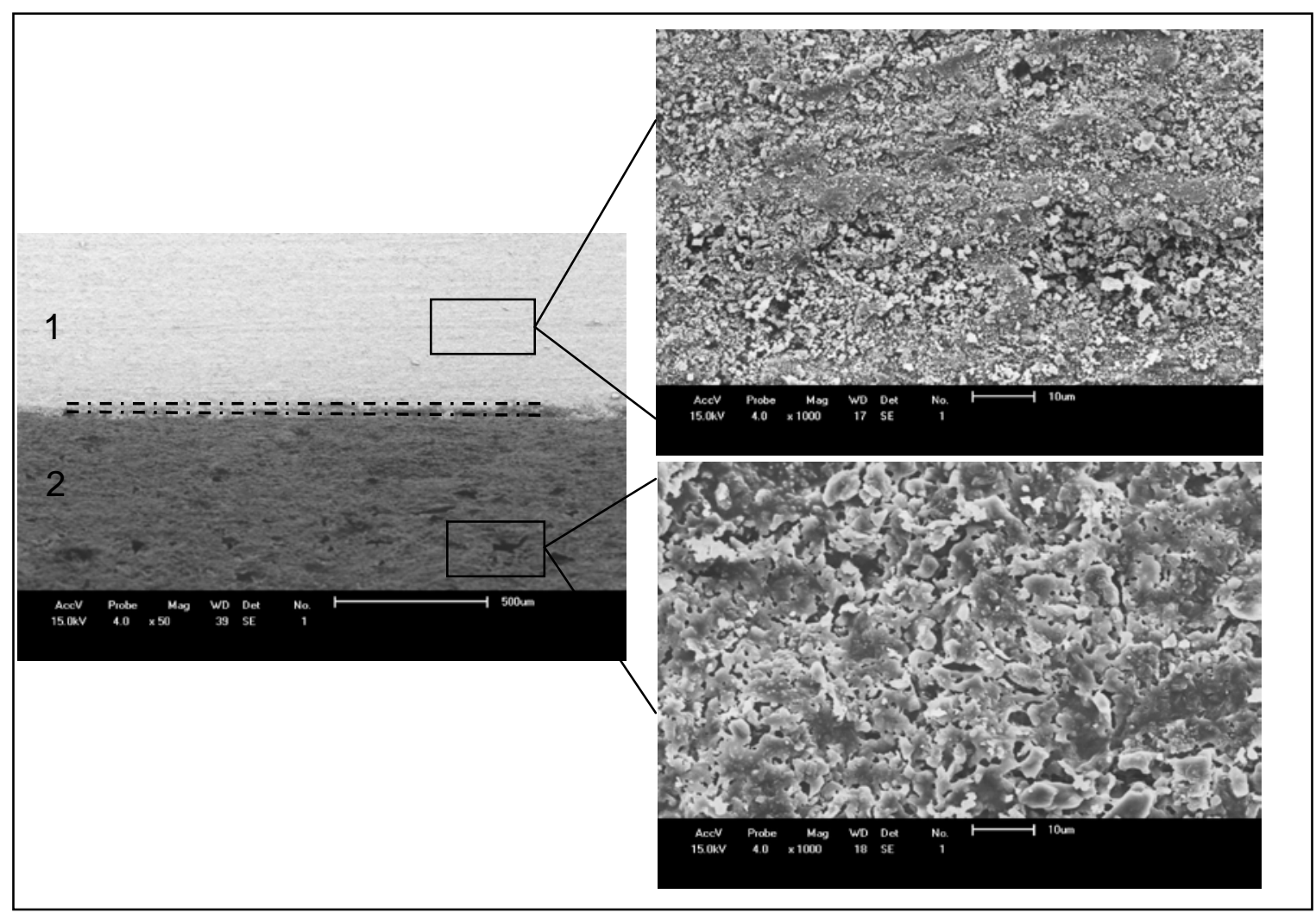

Figura 5: Micrografias da membrana assimétrica obtida no tempo de $5 \mathrm{~s}$ de deposição da dispersão depositada sobre o suporte poroso.

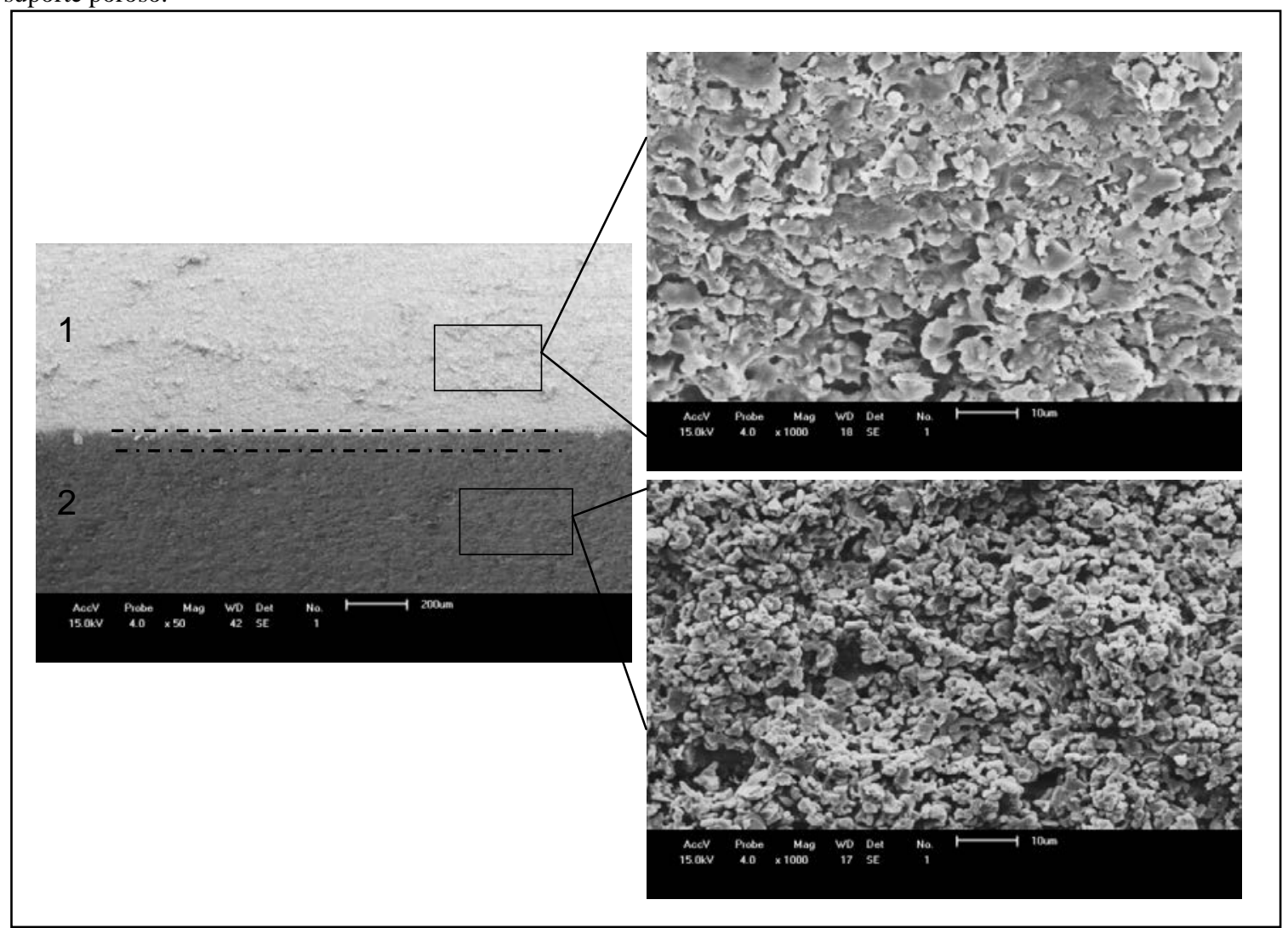

Figura 6: Micrografias da membrana assimétrica obtida no tempo de $10 \mathrm{~s}$ de deposição da dispersão depositada sobre o suporte poroso. 
Os valores do diâmetro médio dos poros em função do volume de intrusão de mercúrio da camada filtrante podem ser vistos na Figura 7.

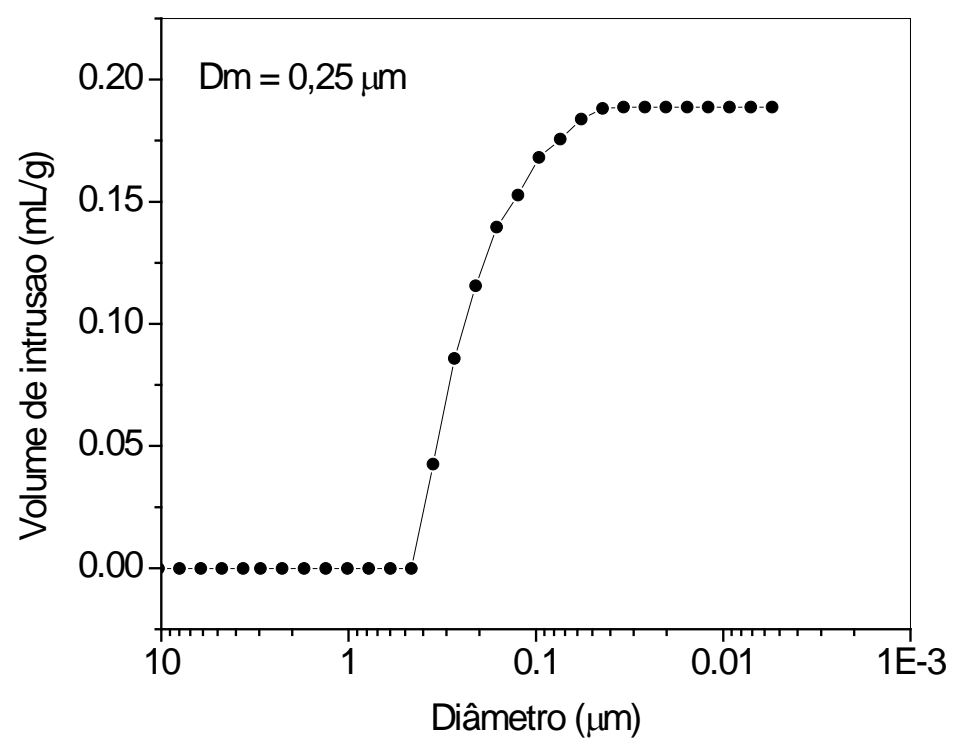

Figura 7: Curva referente à distribuição de poros da camada filtrante da membrana assimétrica.

De acordo com a curva da Figura 7 percebe-se que a camada filtrante da membrana exibe a maioria dos valores para os diâmetros médios dos poros entre 0,4 e 0,04 $\mu \mathrm{m}$ atingindo valor médio de 0,25 $\mu \mathrm{m}$ com porosidade de $35 \%$, classificando-a portanto para aplicações em processos de microfiltração.

O valor alcançado para o diâmetro médio de poro da camada filtrante segue um gradiente adequado para a obtenção da membrana cerâmica assimétrica, ou seja, o diâmetro médio de poro da camada filtrante é menor que o diâmetro médio de poro do suporte com os valores de 0,25 e 0,99 $\mu \mathrm{m}$, respectivamente.

Alguns trabalhos reportados na literatura exibem resultados quanto ao diâmetro médio de poro muito acima do valor exposto nos resultados acima. Dentre esses, podem ser exemplificados a pesquisa realizada por Saffaj et al. [13] onde os autores fizeram uso de argila para obtenção de suportes porosos e atingiram o valor de diâmetro de poros de $11 \mu \mathrm{m}$. Khemakhem et al. [14] fabricaram membranas cerâmicas de microfiltração com argila alcançando poros com cerca de $10 \mu \mathrm{m}$ para aplicação em tratamento de efluentes. Já a pesquisa reportada por Meesaoudi et al. [15] ilustrou um valor mais próximo quando comparado ao valor encontrado na presente pesquisa. Os autores citados atingiram 0,16 $\mu \mathrm{m}$ para o diâmetro médio de poro para membranas também obtidas com argila.

Conforme descrito na literatura [16], a porosidade final da membrana e o tamanho médio de poro dependem, entre outros fatores, do material, da granulometria do pó e da temperatura de sinterização, ou seja, a complexa morfologia para montagem da membrana assimétrica não pode ser justificada com base em uma caracterização apenas, mas sim, com um resultado justificando o outro.

\section{DISCUSSÃO}

A demanda na produção de membranas cerâmicas cresce anualmente devido essas poderem ser aplicadas nos mais diversos ramos científicos e industriais. A literatura tem reportado resultados significativos para a produção de membranas assimétricas produzidas com óxidos como zircônia [1]], titânia [1ㅇ] e alumina [19, 20] entretanto, a presente pesquisa ilustrou resultados compatíveis fazendo-se uso de um material natural como matéria-prima, no caso, argila. A facilidade de obtenção, de preparo e o baixo custo desse material justificam a produção da membrana assimétrica que atingiu diâmetro de poro e porosidade voltada para aplicações nos variados processos de microfiltração, viabilizando, dessa forma, a pesquisa realizada.

\section{CONCLUSÕES}

Diante dos resultados apresentados conclui-se que a composição (90-10)\% de alumina comercial-bentonita é satisfatória para obtenção de suportes porosos tubulares, atingindo diâmetro médio de poro de 0,99 $\mu \mathrm{m}$ e 
39\% de porosidade. A dispersão de argila é viável para obtenção da camada filtrante de membranas assimétricas, esta apresentando melhor morfologia superficial quando depositada no tempo de $10 \mathrm{~s}$ sobre o suporte, alcançando diâmetro médio de poro de $0,25 \mu \mathrm{m}$ e porosidade de $35 \%$, sendo classificada, portanto, para aplicações em processos de microfiltração.

\section{AGRADECIMENTOS}

Os autores deste trabalho agradecem a CAPES pelo apoio financeiro.

\section{BIBLIOGRAFIA}

[1] PALACIO, L., BOUZERDI, Y., OUAMMOU, M., et al., "Ceramic membranes from Moroccan natural clay and phosphate for industrial water treatment”, Desalination, v. 245, n. 1-3, pp. 501-507, Set. 2009.

[2] ZAWRAH, M. F., KHATTAB, R. M., GIRGIS, L. G., et al., "Effect of CTAB as a foaming agent on the properties of alumina ceramic membranes”, Ceramics International, v. 40, n. 4, pp. 5299-5305, maio. 2014.

[3] KHEMAKHEM, S., AMAR, R. B., "Modification of Tunisian clay membrane surface by silane grafting: Application for desalination with Air Gap Membrane Distillation process Colloids and Surfaces A", Physicochemical and Engineering Aspects, v. 387, n. 1-3, pp. 79-85, Ago. 2011.

[4] KHEMAKHEM, S., LARBOT, A., AMAR, R. B., "Study of performances of ceramic microfiltration membrane from Tunisian clay applied to cuttlefish effluents treatment”, Desalination, v. 200, n. 1-3, pp. 307-3091, Nov. 2006.

[5] VERCAUTEREN, S., VAYER, M., DAMME, H. V., et al., "The preparation and characterization of ceramic membranes with a pillared clay top layer. Colloids and Surfaces A", Physicochemical and Engineering Aspects, v. 138, n. 2-3, pp. 367-376, Jul. 1998.

[6] BURGGRAAF, A. J., COF, L. "General overview, trends and prospects", Membrane Science and Technology, v. 4, n.1, p. 1-20, 1996.

[7] SILVA, M. C., LIRA, H. L., SILVA, F. N. S., et al. "Utilização de Matéria-prima Natural de Baixo Custo na Obtenção de Membrana Cerâmica Assimétrica Tubular para Aplicação em Processos de Microfiltração”, Ceramica Industrial, v. 19, n. 4, Julho/Agosto, 2014.

[8] SILVA, L. L. O., VASCONCELOS, D. C. L., NUNES, E. H. M., et al., "Processing, structural characterization and performance of alumina supports used in ceramic membranes", Ceramics International, v. 38, n. 3, pp. 1943-1949, abril. 2012.

[9] CHEONG, H., CHO, W. S., HA, J. S., et al., "Structural evolution of alumina membrane prepared on an alumina support using a sol-gel method", Journal of Alloys and Compounds, v. 290, n. 1-2, pp. 304-309, ago. 1999.

[10] BARMA, S., MANDAL, B., "Effects of sintering temperature and initial compaction load on alphaalumina membrane support quality”, Ceramics International, v. 40, n. 7, pp. 11299-11309, ago. 2014.

[11] DONG, Y., LIN, B., XIE, K., et al., "Cost-effective macro-porous mullite-corundum ceramic membrane supports derived from the industrial grade powder", Journal of Alloys and Compounds, v. 477, n. 1-2, pp. 350-356, maio. 2009.

[12] HU, X., YU, J., SONG, J., et al., "Toward low-cost Pd/ceramic composite membranes for hydrogen separation: A case study on reuse of the recycled porous $\mathrm{Al}_{2} \mathrm{O}_{3}$ substrates in membrane fabrication", International Journal of Hydrogen Energy, v. 36, n. 24, pp. 15794-15802, dez. 2011.

[13] SAFFAJ, N., PERSIN, M., Y., OUNSI, S. A., et al., "Elaboration and characterization of microfiltration and ultrafiltration membranes deposited on raw support prepared from natural Moroccan clay: Application $\mathrm{t} o$ filtration of solution containing dyes and salts” Applied Clay Science, v. 31, n. 1-2, pp. 110-119, jan. 2006.

[14] KAKIHANA, M., "Sol-Gel” Preparation of High Temperature Superconducting Oxides", Journal of Sol-Gel Science and Technology. v. 6, n. 1, pp. 7- 55, 1996.

[15] MESSAOUDI, L., LARBOT, A., RAFIM, M., COT, L., "Fabrication of a microfiltration membrane on tubular supports with a Morocco clay”, L' Industrie Céramique \& Verriére, v. 910, n. 1, pp. 831-835, 1995.

[16] HABERT, A. C., BORGES, C. P., NÓBREGA, R., "Processos de Separação por Membranas”. E-Papers Serviços Editoriais, Rio de Janeiro , 2006. 
[17] YIN, W., MENG, B., MENG, X., et al.,"Highly asymmetric yttria stabilized zirconia hollow fibre membranes", Journal of Alloys and Compounds, v. 476, n. 1-2 , pp. 566-570, mai. 2009.

[18] PORTER, J. J., ZHUANG, S., “Microfiltration of sodium nitrate and Direct Red 2 dye using asymmetric titanium dioxide membranes on porous ceramic tubes”, Journal of membrane science, v. 110, n. 1, pp. 119132, fev. 1996.

[19] FIROUZGHALB, H., FALAMAKI, C., "Fabrication of asymmetric alumina membranes: I. Effect of SrO addition on thermal stabilization of transition aluminas," Materials Science and Engineering: $B$, v. 166, n. 2, pp.163-169, jan. 2010.

[20] PENGLEE, K., MATTIA, D., "Monolithic nanoporous alumina membranes for ultrafiltration applications: Characterization, selectivity-permeability analysis and fouling studies”, Journal of Membrane Science, v. 435, n.1, pp. 52-61, maio. 2013. 